\title{
Identification of cryptic stator subunits from an apicomplexan ATP synthase
}

\author{
Diego Huet ${ }^{1}$, Esther Rajendran², Giel G. Van Dooren², and Sebastian Lourido ${ }^{1,3 *}$ \\ 1 Whitehead Institute for Biomedical Research, Cambridge, Massachusetts, USA. \\ ${ }^{2}$ Research School of Biology, Australian National University, Canberra, Australian Capital Territory, Australia. \\ ${ }^{3}$ Department of Biology, Massachusetts Institute of Technology, Cambridge, Massachusetts, USA. \\ *For correspondence: lourido@wi.mit.edu
}

\section{ABSTRACT}

The mitochondrial ATP synthase is a macromolecular motor that uses the proton gradient to generate ATP. Proper ATP synthase function requires a stator linking the catalytic and rotary portions of the complex. However, sequence-based searches fail to identify genes encoding stator subunits in apicomplexan parasites like Toxoplasma gondii or the related organisms that cause malaria. Here, we identify 11 previously unknown subunits from the Toxoplasma ATP synthase, which lack homologs outside the phylum. Hidden Markov modeling suggests that two of them-ICAP2 and ICAP18 - share distant homology with mammalian stator subunits. Our analysis shows that both proteins form part of the ATP synthase complex. Depletion of ICAP2 leads to aberrant mitochondrial morphology, decreased oxygen consumption, and disassembly of the complex, consistent with its role as an essential component of the Toxoplasma ATP synthase. Our findings highlight divergent features of the central metabolic machinery in apicomplexans, which may reveal new therapeutic opportunities.

\section{INTRODUCTION}

The ATP synthase is a highly conserved protein complex found in the plasma membrane of bacteria, the inner membrane of mitochondria, and the thylakoid membrane of chloroplasts. The complex consists of two functionally distinct portions: the hydrophilic $F_{1}$ and the membranebound $F_{0}$ (Walker, 2013). The mechanics of this molecular motor is best understood for the mitochondrial ATP synthases of yeast and mammals. Within their mitochondria, the proton gradient generated by the electron transport chain (ETC) drives the rotation of a ring of $c$ subunits in $\mathrm{F}_{0}$ and of the attached central stalk within $\mathrm{F}_{1}$. This rotation causes the conformational changes in the $\alpha$ and $\beta$ subunits of $F_{1}$ that mediate catalysis of ATP from ADP and inorganic phosphate $\left(P_{j}\right)$ (Jonckheere, Smeitink, \& Rodenburg, 2012). The stator, also known as the lateral stalk, is an essential component of the ATP synthase because it counteracts the rotation of the $a$ and $\beta$ subunits, enabling ATP synthesis (Dickson, Silvester, Fearnley, Leslie, \& Walker, 2006). It is therefore surprising that despite general conservation of the central subunits, the lateral elements of protozoan ATP synthases are structurally diverse and these organisms appear to lack homologs for the stator subunits of yeast and mammals (Lapaille et al., 2010).

The composition of the mitochondrial ATP synthase has mainly been determined from detailed studies of purified Saccharomyces cerevisiae and Bos taurus mitochondria, both members of the eukaryotic clade Opisthokonta. In these two species, the architecture of the ATP synthase is virtually identical, and sequence analysis and proteomics have identified homology for nearly all of the subunits that constitute the mitochondrial ATP synthase (Wittig \& Schägger, 2008). In contrast, recent studies in different protozoan species have reported unique structural and 
functional features in their ATP synthases. Proteomic and biochemical studies have identified several potential stator subunits in Tetrahymena thermophila and Euglena gracilis, although none appear conserved among protists (Balabaskaran Nina et al., 2010; Perez et al., 2014). Similarly, the ATP synthases of algae like Chlamydomonas reinhardtii and Polytomella spp. and kinetoplastids like Trypanosoma brucei lack canonical stator subunits but possess phylumspecific proteins that likely perform that function (Šubrtová, Panicucci, \& Zíková, 2015; van Lis, Mendoza-Hernández, Groth, \& Atteia, 2007).

Apicomplexans comprise a large phylum of obligate intracellular eukaryotic parasites that infect animals and cause significant human mortality and morbidity. Comparative genomics suggests that there is extensive metabolic diversity within this phylum, reflecting the variety of life cycles and host environments the various species have adapted to. Several core subunits of the ATP synthase $(a, \beta, \gamma, \delta, \varepsilon, c$, and OSCP) can be found in most apicomplexan genomes (Vaidya \& Mather, 2009). However, within the Cryptosporidium genus, drastic reductions in mitochondrial metabolism have coincided with preservation of only the a and $\beta$ subunits in certain species (Liu et al., 2016; Makiuchi \& Nozaki, 2014).

Reliance on mitochondrial metabolism can also vary over the course of a parasite's life cycle. For instance, the rapidly replicating forms of $T$. gondii catabolize glucose via the tricarboxylic acid (TCA) cycle (MacRae et al., 2012), whereas slow replicating stages seem to lack a functional TCA cycle and rely on glycolysis of stored amylopectin (Denton, Roberts, Alexander, Thong, \& Coombs, 1996; Uboldi et al., 2015). A comparable metabolic shift seems to occur when T. gondii parasites egress from host cells and rely on oxidative phosphorylation for the majority of their ATP production (MacRae et al., 2012). Plasmodium spp. increase their TCA cycle activity as they transition from the asexual blood stages to the sexual cycle (MacRae et al., 2013). Consequently, deletion of the ATP synthase $\beta$ subunit marginally affects blood stages but is essential for the sexual stages in the mosquito (Sturm, Mollard, Cozijnsen, Goodman, \& McFadden, 2015). Together with pharmacological assays, these results confirm that the apicomplexan ATP synthase is active and essential in at least some life cycle stages (Balabaskaran Nina et al., 2011; Sturm et al., 2015). Direct measurements of ATP synthase activity in T. gondii (Vercesi, Rodrigues, Uyemura, Zhong, \& Moreno, 1998) further suggest that these parasites must employ highly divergent stator subunits to form a functional enzyme.

To improve our ability to functionally characterize the T. gondii genome, our lab previously developed a CRISPR-based genome-wide screen that identified genes contributing to parasite fitness (Sidik et al., 2016). Approximately 360 of these fitness-conferring genes were found to be broadly conserved among apicomplexans, but not other eukaryotes, and most lacked functional annotation; we referred to them as indispensable conserved apicomplexan proteins (ICAPs). Of the 17 ICAPs we determined a subcellular localization for, eight localized to the mitochondrion. This preponderance of ICAPs in the mitochondrion motivated further investigation.

Using unbiased proteomic and distant-homology analyses, we show that two mitochondrial ICAPs_ICAP2 and ICAP18 - share homology with the ATP synthase stator subunits of opisthokonts. We show that both proteins interact with known and novel components of the ATP synthase, consistent with their role as putative subunits of the complex. Conditional depletion of ICAP2 leads to aberrant mitochondrial morphology and the disassembly of the ATP synthase complex, consistent with its proposed role in the stator. Our study reveals new information regarding the unconventional apicomplexan stator and provides a perspective on the divergent nature of the ATP synthase in this important group of pathogens. 


\section{RESULTS}

\section{An indispensable conserved apicomplexan protein shares distant homology with bovine and fungal ATP synthase b subunits.}

ICAP2 (TGME49_231410) was originally described in our T. gondii genome-wide screens as one of the eight indispensable conserved apicomplexan proteins (ICAPS) that localized to the mitochondrion of the parasite (Sidik et al., 2016). These mitochondrial ICAPs lacked functional annotation, identifiable protein domains or homology to sequences outside the apicomplexan phylum. We therefore used the protein function and structure prediction server HHPRED to search for distant homologs on the basis of profile hidden Markov models and secondary structure predictions (Zimmermann et al., 2017). Intriguingly, ICAP2 was predicted to contain a region of potential homology to a stator component of a mammalian ATP synthase, the bovine b subunit (BtATPb; 97.8\% probability), despite extremely low sequence identity between the two ( 24\%; Figure 1A). Subcellular localization and topology predictions support the presence of a mitochondrial targeting signal on ICAP2 as well as two transmembrane domains, similar to BtATPb. Despite the similarities, ICAP2 was predicted to be nearly twice as long as BtATPb. Unexpectedly, the analysis also predicted the presence of a putative calcium-binding domain in the N-terminal portion of ICAP2 (Figure 1A; 74.5\% probability). ICAP2 homologs were present in most apicomplexan genomes with the exception of some Cryptosporidium spp. and Gregarina niphandrodes, which lack most known ATP synthase subunits (Vaidya \& Mather, 2009). An ICAP2 homolog was also found in Chromera velia, a free-living autotrophic relative of apicomplexans; and no additional related sequences were found outside these groups. An alignment of the ICAP2 homologs show extended regions of sequence identity including the putative calcium-binding domain and the region of similarity to BtATPb (Figure 1B and Figure S1). The alignment confirmed the extensive conservation of ICAP2 throughout the phylum (Figure 1C).

Figure 1. ICAP2 is a conserved apicomplexan protein with distant similarity to ATP synthase $b$ subunits. (A) Schematic of the Bos taurus ATP synthase $b$ subunit (BtATPb) and ICAP2 showing the mitochondrial targeting signal (MTS), transmembrane domains (TMs), and the putative $\mathrm{Ca}^{2+}$-binding domain of ICAP2 . The position of the region of similarity (dark blue) is numbered according to the amino acid sequence. (B) Percent identity plot of the aligned ICAP2 homologs from diverse apicomplexans (shown in Figure S1). Mean identity within a rolling window of ten residues is plotted. Domains are colored according to the schematic (A) following the positions of the $T$. gondii residues in the alignment. (C) Neighbor-joining tree showing the phylogenetic relationships of ICAP2 homologs analyzed in B. Bootstrap values for 10,000 trials are displayed. (D) Immunoblot showing expression of Ty-

A

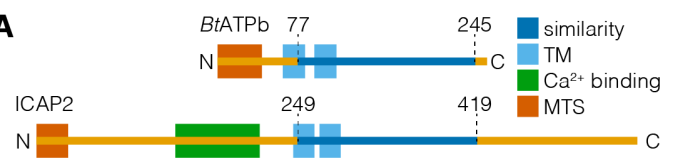

C

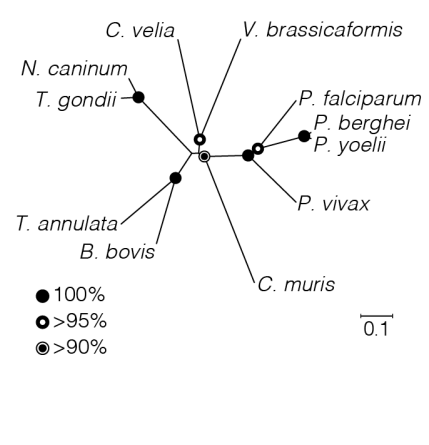

D

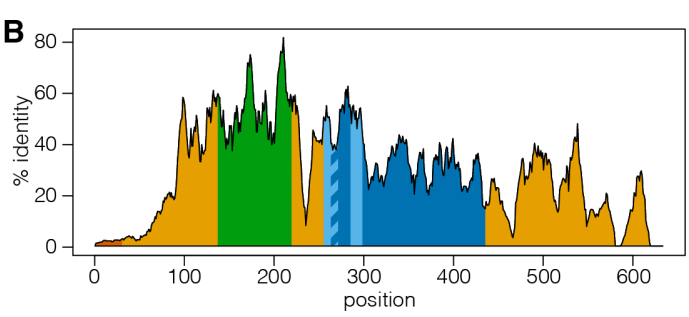

E

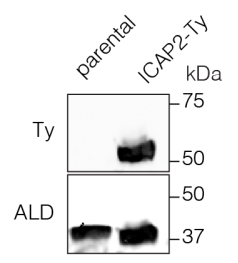

HSP70

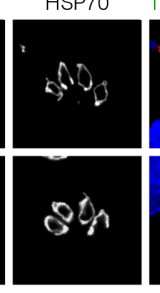

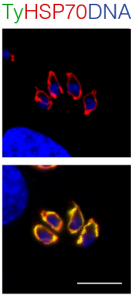
tagged ICAP2 from its endogenous locus in the ICAP2-Ty strain. ALD serves as a loading control. (E) Intracellular parasites from the parental and ICAP2-Ty strains fixed and stained for Ty (green), HSP70 (red) and DAPI (blue). Scale bar is10 $\mu \mathrm{m}$. 
Using CRISPR-mediated endogenous tagging, we introduced a C-terminal Ty epitope into the ICAP2 locus of the TATi $\triangle K U 80$ strain of T. gondii (Sheiner et al., 2011). A clonal strain was generated by limiting dilution, and expression of ICAP2-Ty at the predicted molecular weight was confirmed by immunoblotting (Figure 1D). Localization of ICAP2 was determined by immunofluorescence where the protein colocalized with the mitochondrial marker HSP70 (Pino et al., 2010) (Figure 1E), in agreement with previous results (Sidik et al., 2016).

\section{ICAP2 associates with known and novel components of the ATP synthase.}

Based on distant homology of ICAP2 to BtATPb and its mitochondrial localization, we sought to determine whether the protein interacts with the ATP synthase of T.gondii. We immunoprecipitated ICAP2 from the ICAP2-Ty strain using an antibody against Ty and then eluting the bound proteins under native conditions with excess Ty peptide. The parental strain was used as a specificity control. The input, unbound and eluted fractions were resolved by SDS-PAGE and subjected to immunoblotting. This demonstrated that ICAP2 co-immunoprecipitated with the $\beta$ subunit of the ATP synthase, but not with an irrelevant cytosolic protein (ALD; Figure 2A). The total protein in the eluates was further visualized by silver stain, showing several proteins that coimmunoprecipitated with ICAP2-Ty (Figure 2B). We excised eight different sections of the gel and analyzed them by mass spectrometry. This analysis identified nearly all the annotated ATP synthase subunits found in $T$. gondii except for the $c$ subunit, revealing that ICAP2 physically interacts with the ATP synthase complex.

Among the proteins immunoprecipitated with ICAP2, was the previously uncharacterized TGME49_268830, which we referred to as ICAP18 because it was part of our original ICAP list, although we were unable to tag it in the initial study (Sidik et al., 2016). HHPRED suggested that ICAP18 is distantly homologous to the second major component of the bovine stator, the

Figure 2. ICAP2 associates with known components of the ATP synthase. (A) Immunoprecipitation of Ty from the parental and ICAP2-Ty strains. Immunoblot for Ty, $F_{1} \beta$ and ALD in the input, unbound and eluted fractions. (B) Following Ty immunoprecipitation, the ICAP2-Ty eluted fraction was separated by SDS-PAGE and proteins were visualized by silver staining. The eight gel fractions analyzed by mass spectrometry are labeled according to the known ATP synthase subunits identified in them, along with ICAP2 and ICAP18. Asterisks indicate bands where no known ATP synthase subunits could be identified. (C) Schematic representation of the Bos taurus ATP synthase $d$ subunit (BtATPd) and ICAP18 showing the MTS, TMs and the region of similarity (dark blue). (D) Percent identity plot of the aligned ICAP18 homologs from diverse apicomplexans (shown in Figure S2). Mean identity within a rolling window of ten residues is plotted. Domains are colored according to the diagram $(\mathrm{C})$, and numbered according to the positions of the T. gondii residues in the alignment. (E) Neighbor-joining tree showing the phylogenetic relationships of the ICAP18 homologs analyzed in D. Bootstrap values for 10,000 trials are displayed. (F) Intracellular parasites from parental and ICAP18-Ty strains fixed and stained for Ty (green), HSP70 (red), and DAPI (blue). Scale bar is $10 \mu \mathrm{m}$.
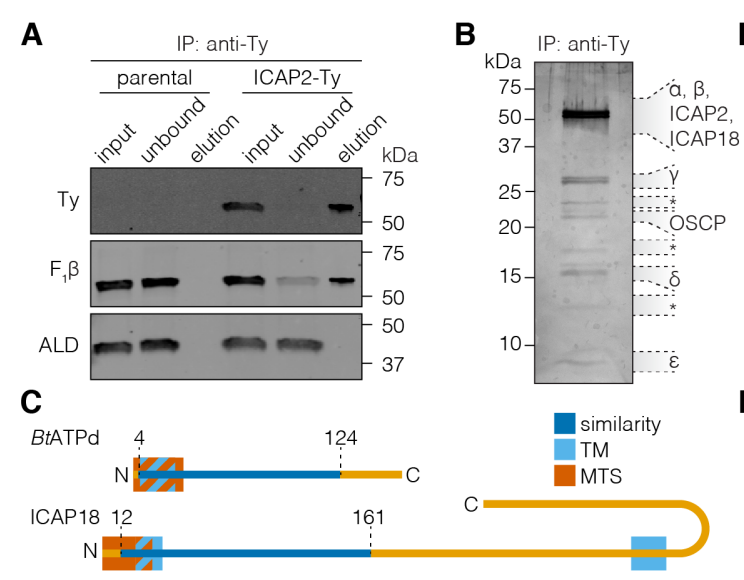

D

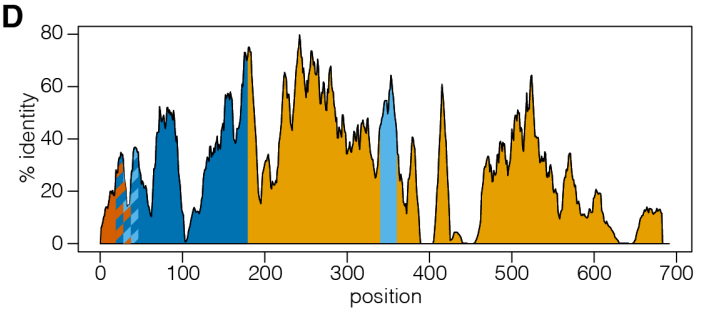

$\mathbf{F}$
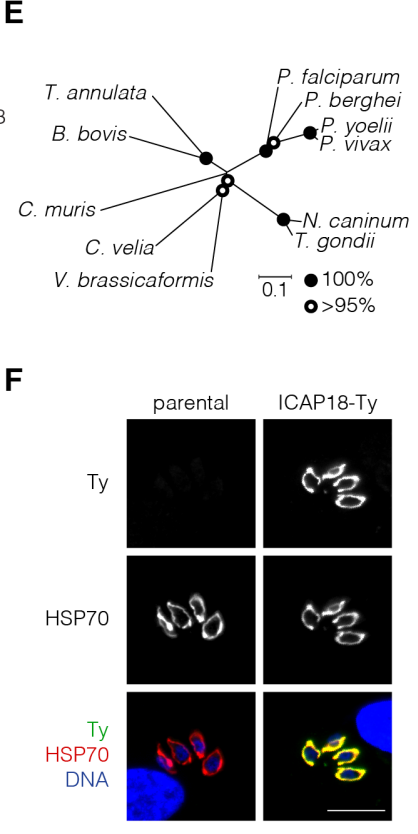

BtATPd

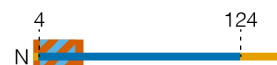

CAP18 12

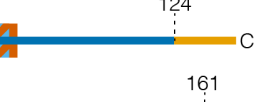

$95 \%$ 
d subunit of the ATP synthase (BtATPd; $96.17 \%$ probability), despite only $17 \%$ sequence identity between the two proteins. Homologs of ICAP18 were present in apicomplexan and chromerid genomes, with the exception of organisms with reduced mitochondrial metabolism, mirroring the distribution of ICAP2. No additional sequences related to ICAP18 were found outside these groups. Similarity to BtATPd was restricted to the N terminus of ICAP18 where mitochondrial targeting signals and a transmembrane domain (TM) are predicted for both proteins (Figure 2C). ICAP18 additionally possesses a long C-terminal extension that contains a second predicted TM domain. All of these sequence features showed significant conservation in the alignment of the apicomplexan and chromerid ICAP18 homologs (Figure 2D and E, and Figure S2).

To further study the protein, we tagged the endogenous ICAP18 locus using CRISPR to introduce a Ty epitope tag by homologous recombination. The tagged strain (ICAP18-Ty) was cloned by limiting dilution. Immunofluorescence confirmed the mitochondrial localization of ICAP18-Ty by colocalization with the HSP70 mitochondrial marker (Figure 2F). Taken together, these observations indicate that ICAP2 and ICAP18 are putative stator subunits of the T. gondii ATP synthase.

\section{A novel array of proteins is associated with the T. gondii ATP synthase}

To directly test the association between ICAP18 and the ATP synthase, we repeated the native Ty immunoprecipitation using ICAP18-Ty as the bait. This confirmed the interaction between ICAP18 and the $\beta$ subunit of the ATP synthase $\left(F_{1} \beta\right.$; Figure 3A $)$. Comparison of eluates from the ICAP2-Ty and ICAP18-Ty strains showed comparable protein patterns when visualized by silver stain (Figure 3B). The lack of signal from the parental strain confirmed the specificity of immunoprecipitation.

To identify additional proteins associated with the T. gondii ATP synthase, we repeated the mass spectrometry analysis three times for ICAP2-Ty, and once for ICAP18-Ty, identifying a total of 209 proteins in aggregate (Figure S3 and Table S1). To determine which proteins were most likely components of the ATP synthase, we filtered the results based on several criteria (Figure 3C). First, we predicted that novel subunits would follow the same pattern of phylogenomic conservation as ICAP2, ICAP18 and most of the known ATP synthase subunits. We therefore performed BLAST (Boratyn et al., 2013) searches against each candidate to determine whether homologs could be found in $P$. falciparum, $C$. velia and $C$. muris (E value $<10^{-5}$ ) but not $C$. parvum (E value $>10^{-5}$ ). Outside of the known subunits, only 16 proteins exhibited this pattern of conservation. The known ATP synthase subunits are also synchronously expressed along the cell cycle of T. gondii (Behnke et al., 2010). We therefore compared the expression pattern of each gene to the mean expression pattern of the known ATP synthase subunits by calculating the Spearman's correlation coefficient $\left(r_{s}\right)$. Of the 16 genes above, nine were co-regulated with the known subunits $\left(r_{s}>0.85\right.$; Figure 3D and Table S1). All nine proteins, as well as all of the known subunits, were predicted to contribute to parasite fitness in our genome-wide screens (Sidik et al., 2016). The final list of novel subunits contained eight of the nine proteins. TGME49_226970 was excluded from the final list because it was clearly the ribosomal protein RPS11 (Sun et al., 2015). TGME49_215610 was ultimately included because it fit all the criteria except for coregulation, which could not be evaluated due to a lack of expression data.

Two proteins identified by mass spectrometry did not meet our criteria, but are nonetheless worth mentioning. TGME49_215350 was only identified in a single mass spectrometry experiment, and failed all of the criteria mentioned above, yet HHPRED suggested it is distantly 
A

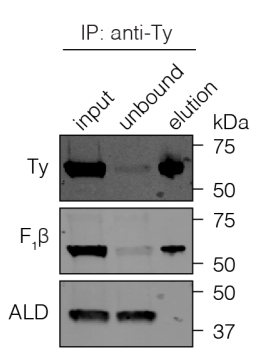

B

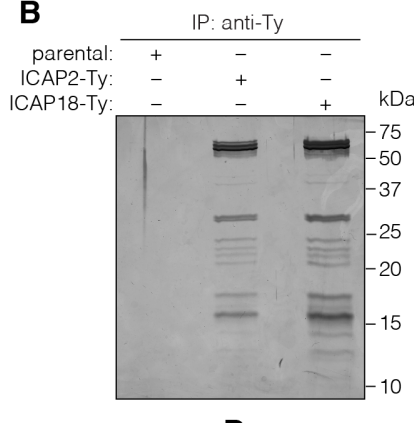

D

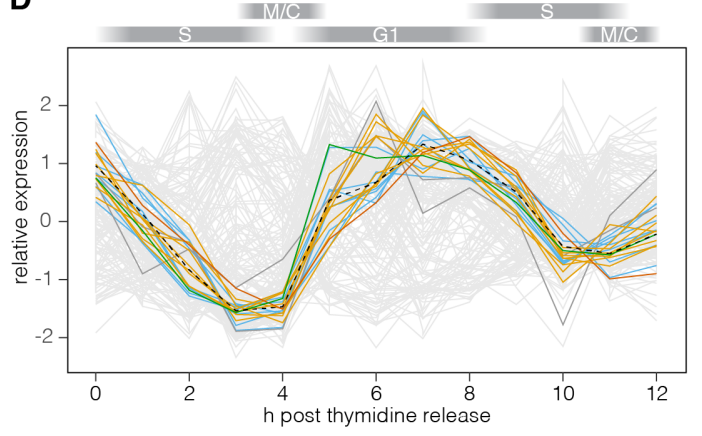

C

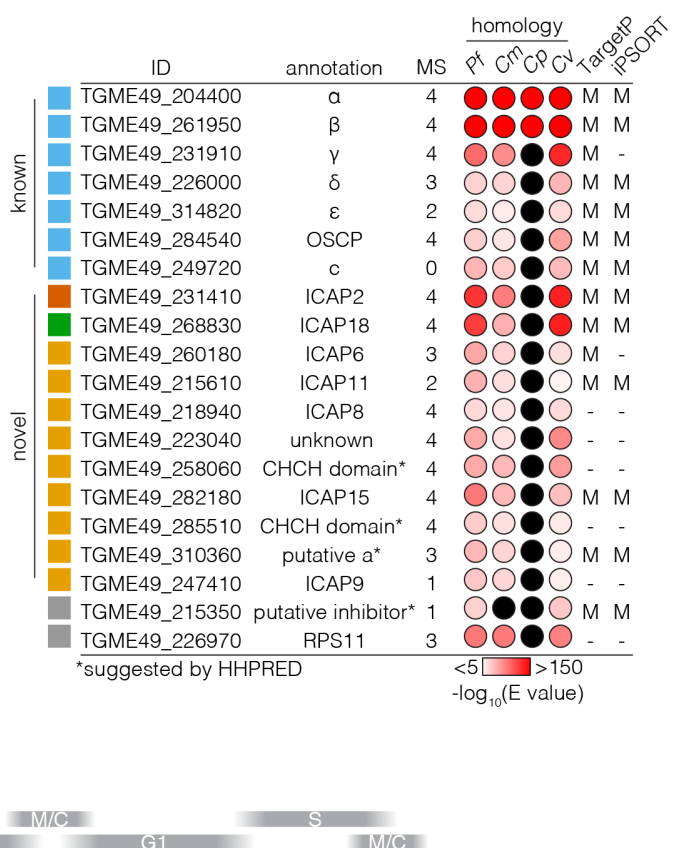

Figure 3. The $T$. gondii ATP synthase associates with proteins conserved among apicomplexans. (A) Immunoprecipitation of Ty from the ICAP18-Ty strain. Immunoblot for $T y, F_{\beta} \beta$ and ALD in the input, unbound and eluted fractions. (B) Following Ty immunoprecipitation, the eluates of the parental, ICAP2-Ty and ICAP18-Ty strains were separated by SDSPAGE and visualized by silver staining. (C) Table showing the known and novel ATP synthase subunits, including ICAP2 (red) and ICAP18 (green). The table lists the proposed annotation of each gene and the number of times each protein was found in the MS experiments. Proteins in grey represent candidates that did not meet the analysis criteria, or were clear contaminants. The homology indicates the BLAST expected value (E value) between each T. gondii protein sequence and that of its closest match in Plasmodium falciparum (Pf), Cryptosporidium muris $(\mathrm{Cm})$, Cryptosporidium parvum $(\mathrm{Cp})$, or Chromera velia $(\mathrm{Cv})$. Cases in which a close match could not be identified ( $\mathrm{E}$ value $<0.0001)$ are indicated in black. The predicted subcellular localization ('M' for mitochondrial or '-' for another location) was determined using TargetP and iPSORT. (D) Relative expression pattern of known ATP synthase subunits (blue), ICAP2 (red), ICAP18 (green), and other novel associated proteins (yellow). Proteins that did not meet the analysis criteria are colored grey. The dotted line represents the mean relative expression of the known ATP synthase subunits. Cellcycle stages are indicated above the plot.

homologous to the yeast ATP synthase inhibitor (95.6\% probability). In contrast, cytochrome $c_{1}$ (TGME49_246540), a component of complex III of the ETC, was identified in the four mass spectrometry experiments and met all of our criteria except for being marginally below the coregulation cutoff.

All of the subunits in our high-confidence list were previously considered among the 360 indispensable conserved apicomplexan proteins (ICAPS) identified in our genome-wide screen. As part of our previous analysis, we Ty-tagged five of these novel subunits (ICAP6, ICAP8, ICAP9, ICAP11, and ICAP15), and showed that all of them localized to the mitochondrion. In other words, of the eight ICAPs previously localized to the mitochondrion, six are associated with the ATP synthase complex.

\section{ICAP2 is necessary for T. gondii growth}

To further investigate the function of ICAP2, we generated a conditional knockdown strain (ICAP2-Ty cKD) using the recently developed U1-snRNP gene silencing strategy (Pieperhoff et al., 2015). This approach relies on the modification of the 3' end of a gene of interest in a DiCreexpressing strain to introduce a floxed synthetic 3' end followed by U1-binding sites. Activation of DiCre with rapamycin leads to recombination, which positions the U1 sites as part of the 
transcript and leads to mRNA degradation. Using CRISPR/Cas9, we modified the 3' end of ICAP2 accordingly, including an in-frame Ty tag and a hypoxanthine phosphoribosyltransferase (HXGPRT) selectable marker (Figure 4A). A $2 \mathrm{~h}$ treatment of the cKD strain with rapamycin was sufficient to observe complete ICAP2-Ty degradation $48 \mathrm{~h}$ later by immunoblot (Figure 4B) and immunofluorescence (Figure 4C).

To assess viability of parasites following ICAP2 depletion, we compared the number of plaques formed by parasites following a $2 \mathrm{~h}$ rapamycin treatment to parasites treated with a vehicle control (DMSO). No plaques were observed upon ICAP2 depletion (Figure 4D) corroborating the protein's role in parasite fitness. We also measured intracellular growth by counting the number of parasites per vacuole at different time points following the rapamycin pulse. We consistently observed fewer large vacuoles in the rapamycin-treated cKD when compared to the paired controls, indicative of impaired cellular replication in ICAP2-depleted parasites (Figure 4E).

A

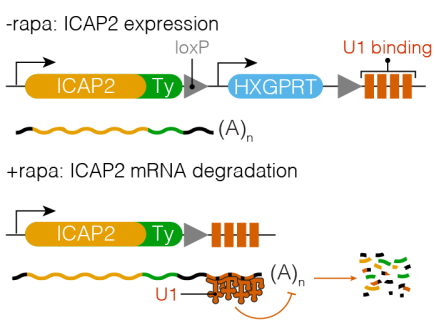

B

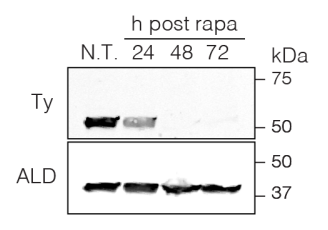

D

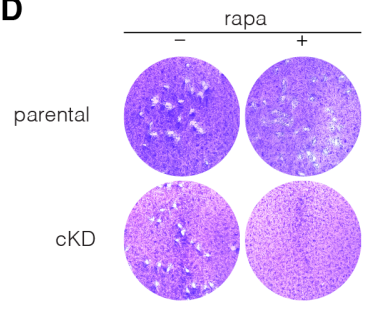

C

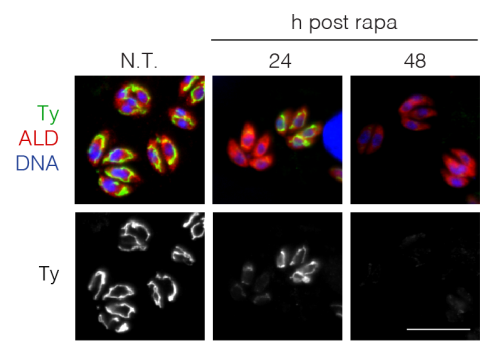

E

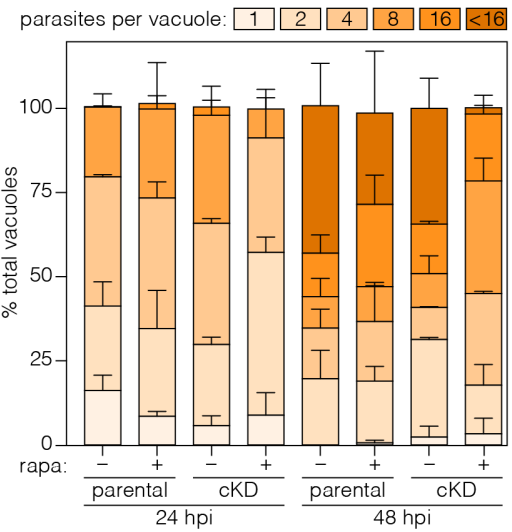

Figure 4. Functional characterization of ICAP2. (A) Schematic of the ICAP2 locus in the ICAP2-Ty CKD strain indicating the U1-mediated mRNA degradation following the rearrangement caused by a brief pulse of rapamycin (rapa). (B) Immunoblot of the ICAP2-Ty cKD strain monitoring degradation of ICAP2-Ty at different time points following a $2 \mathrm{~h}$ pulse with rapa. ALD serves as a loading control. (C) At different time points following treatment with rapa or vehicle (N.T.), intracellular ICAP2-Ty cKD parasites were fixed and stained for Ty (green), ALD (red), and DAPI (blue). Scale bar is $10 \mu \mathrm{m}$. (D) Plaque assay of the parental and ICAP2Ty CKD strains after treatment with rapa or a vehicle control (DMSO). (E) The parental and ICAP2-Ty cKD strains were pulsed with rapa or a vehicle control $24 \mathrm{~h}$ prior to passaging. Samples were fixed 24 or $48 \mathrm{~h}$ post infection (hpi) and stained for Ty and ALD. The distribution of parasites per vacuole was determined. Bars represent mean \pm SD. for $n=2$ independent experiments. At least 150 vacuoles were counted per condition.

\section{Loss of ICAP2 triggers aberrant mitochondrial morphology}

In yeast, ATP synthase dysfunction has been linked to aberrant mitochondrial morphology, reduced cristae formation, matrix swelling, and organellar fragmentation (Youle \& van der Bliek, 2012). We therefore tested the impact of ICAP2 depletion on mitochondrial morphology. Apicomplexan parasites possess a single mitochondrion that often encircles the nucleus of intracellular parasites (Ovciarikova, Lemgruber, Stilger, Sullivan, \& Sheiner, 2017). Immunofluorescence microscopy of ICAP2-depleted parasites indicated that mitochondria lose their characteristic lasso shape, and appear to fragment 24-48 $\mathrm{h}$ after rapamycin treatment (Figure 5A). To rule out any artifacts from fixation, we further investigated this phenotype by live microscopy using parental and ICAP2-Ty cKD strains expressing GFP fused to the mitochondrial targeting signal of SOD2 (SOD2-GFP), as previously described (Pino et al., 2007). Both strains also express the inner membrane complex protein IMC1 fused to 
TdTomato, allowing us to exclusively focus on non-dividing parasites, and thereby exclude from our analysis the known mitochondrial dynamics that occur during the cell division (Nishi, $\mathrm{Hu}$, Murray, \& Roos, 2008). We measured mitochondrial volume in vacuoles containing one or two parasites following treatment with rapamycin or a vehicle control. Volume calculations were performed using Mitograph, an image processing method that enables estimates of the volume of three-dimensional organelles (Viana, Lim, \& Rafelski, 2015) (Figure 5B). In addition to the observed fragmentation, the mitochondrial volume of ICAP2-depleted parasites was significantly reduced at 24 and $48 \mathrm{~h}$ after rapamycin treatment compared to untreated controls or the treated parental strain (Figure 5C).

Mutations in the $b$ subunit of yeast ATP synthase lead to a loss of cristae and outer membrane involutions that give mitochondria an onion-like morphology (Bornhövd, Vogel, Neupert, \& Reichert, 2006; Weimann, Vaillier, Salin, \& Velours, 2008). We therefore examined the ultrastructure of mitochondria in ICAP2-deficient parasites by transmission electron microscopy. Sections containing mitochondrial structures were blinded and randomized to measure mitochondrial area and quantify the number of cristae (Figure 5D-F). We analyzed 73 sections for the control and 92 for the ICAP2-deficient parasites. The distribution of mitochondrial areas was comparable in the presence or absence of ICAP2, likely indicating similar thickness of the mitochondrial structures (Figure 5E). However, because the sections had been pre-selected to contain mitochondria, the similar areas do not reflect the general abundance or volume of the mitochondria within each sample. Notably, the number of cristae was significantly reduced upon ICAP2 depletion (Figure 5F), and in some cases the matrix appeared swollen. Taken together, these results demonstrate the profound effects that ICAP2 loss has on the mitochondrial morphology of T. gondii.

A

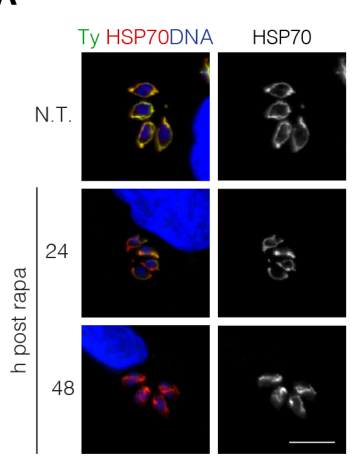

D

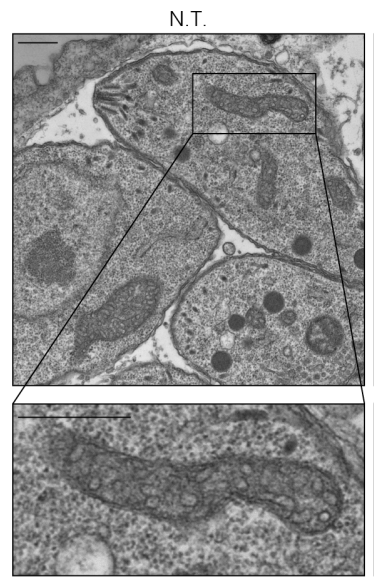

B
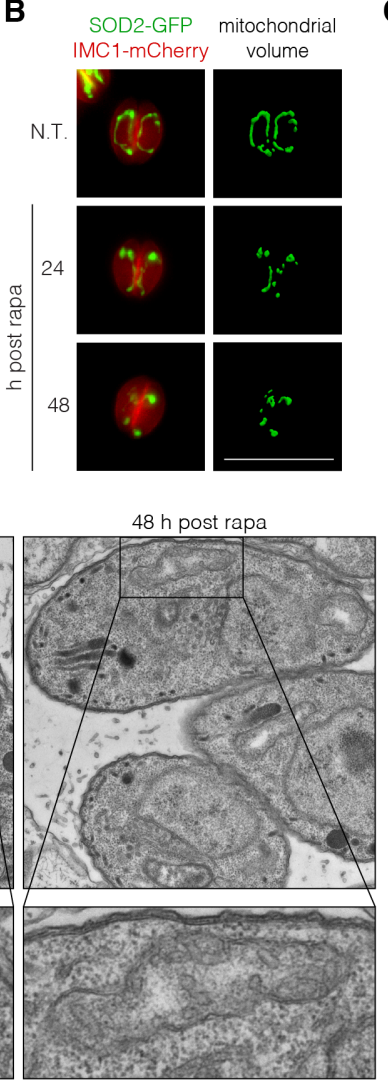

C

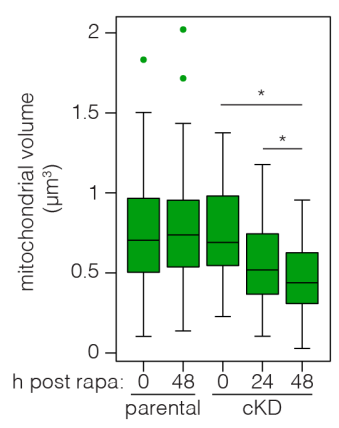

E

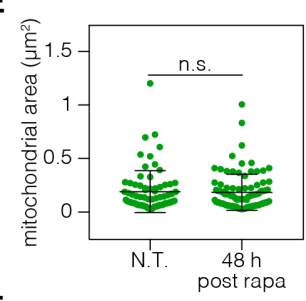

$\mathbf{F}$

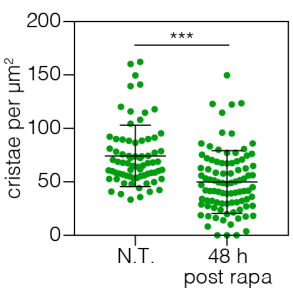

Figure 5. ICAP2 depletion alters mitochondrial morphology.

Mitochondrial morphology in the ICAP2Ty CKD parasites visualized by staining for HSP70 (red), ICAP2-Ty (green), and DAPI (blue), at various time points following a $2 \mathrm{~h}$ pulse with rapa. Scale bar is10 $\mu \mathrm{m}$. (B-C) Changes in mitochondrial volume following ICAP2 depletion. Representative panels displaying the maximum intensity projections and mitochondrial volume from ICAP2-Ty cKD parasites expressing SOD2-GFP and IMC1-TdTomato (B). Live intracellular parasites were imaged following the various treatments. Scale bar is $10 \mu \mathrm{m}$. Quantification of the mitochondrial volume per parasite in vacuoles containing 1 or 2 parasites $(\mathbf{C})$. Boxplot, ${ }^{*} p \leq 0.05$; Student's t-test. (D-F) Electron micrographs of ICAP2-Ty cKD parasites $48 \mathrm{~h}$ after a rapa pulse or treatment with a vehicle control. Insets show representative mitochondria. Scale bars are $500 \mathrm{~nm}$. Blinded analysis of cristae density measured mitochondrial area and the number of cristae per $\mu m^{2}$ within those areas. Mean $\pm S D$, n.s. $p>$ $0.05,{ }^{* * *} p<0.0001$ by a Mann-Whitney test. 


\section{Loss of ICAP2 affects the function and conformation of the ATP synthase}

The ATP synthase dissipates the proton gradient across the mitochondrial inner membrane, thereby increasing the activity of the ETC and leading to higher rates of oxygen consumption. To assess the effect of ICAP2 on ATP synthase activity, we measured the basal mitochondrial oxygen consumption rate (mOCR) in isolated parasites, following different periods of ICAP2 depletion. The basal mOCR of the mutant cells was significantly reduced $72 \mathrm{~h}$ after rapamycin treatment (Figure 6A), indicating a reduction in ETC activity upon depletion of ICAP2. We simultaneously determined the extracellular acidification ratio (ECAR), a measure for parasite metabolic activity (accompanying manuscript by Seidi et al), which was unchanged upon ICAP2 knockdown (Figure S4A), suggesting that the defects we observed in mOCR are not the result of reduced parasite metabolic activity. The upper limit on mOCR imposed by proton transport through the ATP synthase can be relieved using carbonyl cyanide-4 (trifluoromethoxy) phenylhydrazone (FCCP), which acts as a mobile proton carrier that uncouples ETC function from ATP production. Treating cells with FCCP thereby allows us to measure the maximum OCR from which we can calculate the spare mOCR - the difference between basal mOCR (before FCCP addition) and maximum mOCR (after FCCP addition). We observed that the maximal mOCR was not significantly affected by ICAP2 depletion (Figure S4B), and that spare mOCR was significantly increased (Figure 6B). These data are consistent with depletion of ICAP2 leading to defects in ATP synthase function, thereby impairing oxidative phosphorylation in the parasite.

To determine whether ICAP2 plays a role in the stability of the ATP synthase complex-as would be expected of a stator subunit-we analyzed the abundance and migration of the $\beta$ subunit using SDS and blue native (BN) polyacrylamide gel electrophoresis (BN-PAGE). The relative abundance of the $\beta$ subunit did not vary in response to ICAP2 expression, as determined by immunoblot of lysates resolved by SDS-PAGE (Figure 6C). Immunoblots of the same lysates resolved by BN-PAGE showed the $\beta$ subunit participates in a complex of approximately

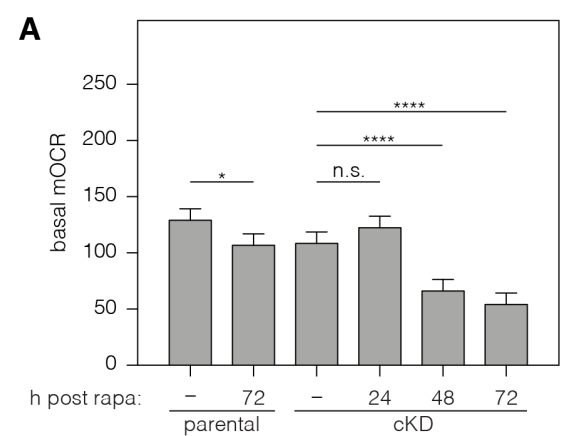

B

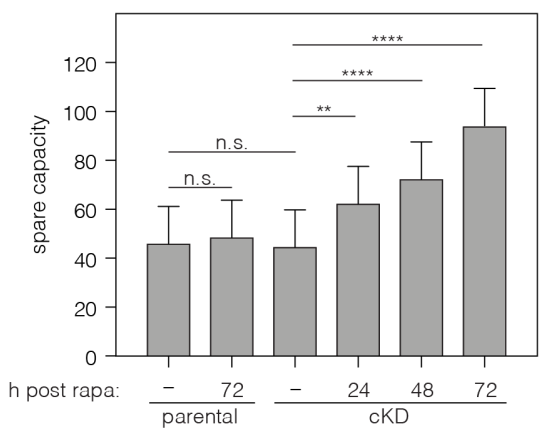

C

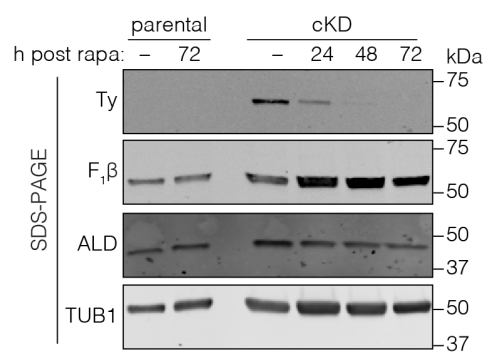

D

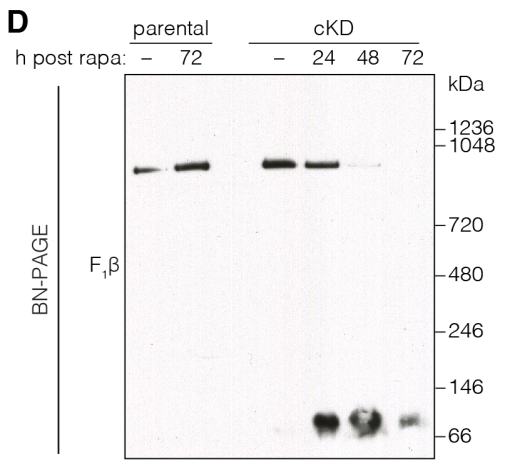

Figure 6. Loss of ICAP2 affects the function and integrity of ATP synthase. (A-B) Mitochondrial oxygen consumption (mOCR in $\mathrm{pmol} / \mathrm{min} / 1.5 \times 10^{6}$ parasites) was determined for the parental and ICAP2-Ty cKD strains at various time points following a $2 \mathrm{~h}$ pulse with rapa. Basal mOCR (A) was compared to the maximum mOCR obtained after treating with the uncoupling agent FCCP to calculate the spare capacity $(\mathbf{B})$. Data represent mean \pm SEM for $n=4$ independent experiments: ** $p$ $<0.005$; ${ }^{* * \star *} p<0.0001$; n.s. not significant; one-way ANOVA followed by Tukey's test. (C-D) Lysates from the parental and ICAP2Ty CKD strains were prepared at various time points following a $2 \mathrm{~h}$ rapa pulse. Lysates were resolved by SDS-PAGE (C) or blue native PAGE (BN-PAGE) and blotted to probe for Ty, $F_{1} \beta$, ALD or TUB. 
$900 \mathrm{kDa}$ in untreated cells. However, upon ICAP2 depletion, the $\beta$ subunit shifted to a much smaller complex, migrating at $\sim 100 \mathrm{kDa}$ (Figure 6D). A significant proportion of the total $\beta$ subunit could be observed in this lower complex for the cKD as early as $24 \mathrm{~h}$ after rapamycin treatment, and increasing thereafter. These observations are consistent with the dissolution of the ATP synthase complex upon loss of ICAP2, similar to what has been observed in yeast and mammalian mutants lacking stator subunits (He, Carroll, Ding, Fearnley, \& Walker, 2017; Kane, Youngman, Jensen, \& van Eyk, 2010; Soubannier et al., 2002). Taken together, these results show that ICAP2 is required for the proper assembly and function of the T. gondii ATP synthase.

\section{DISCUSSION}

The elegant rotary mechanism of the mitochondrial ATP synthase requires a stator to impart the conformational changes on $F_{1}$ that drive ATP catalysis. It has therefore been a long-standing conundrum that most apicomplexan genomes appear to code for only the core subunits of the ATP synthase but none of the stator subunits. Here, we propose that ICAP2 (TGME49_231410) and ICAP18 (TGME49_268830) fulfill the roles of the stator $b$ and $d$ subunits, respectively. Both proteins localize to the mitochondrion of $T$. gondii and share distant homology with the respective subunits of the bovine ATP synthase, despite their unusual length and domain composition. Immunocapture of these putative subunits showed that they stably interact with the ATP synthase complex, along with at least nine other proteins that are conserved among apicomplexans. Moreover, conditional knockdown of ICAP2 leads to abnormal mitochondrial morphology, slow replication and parasite death. ICAP2 was also necessary for the activity of the ATP synthase, and its absence caused a reduction in oxygen consumption by the mitochondrion and disassembly of the complex. Our results reveal highly divergent aspects of the apicomplexan ATP synthase and demonstrate their importance for parasite viability.

We previously identified ICAP2 as one of eight novel mitochondrial proteins, which we localized because of their roles in parasite fitness, a lack of functional domains, and conservation among apicomplexans (Sidik et al., 2016). Pattern hidden Markov models and secondary structure predictions_implemented in HHPRED or Phyre2 (Mezulis, Yates, Wass, Sternberg, \& Kelley, 2015; Zimmermann et al., 2017)_first hinted at the distant homology between ICAP2 and the $b$ subunit of the mammalian ATP synthase. These methods have also helped us assign putative functions to other subunits we isolated as part of the ATP synthase complex of $T$. gondii, including the putative $d$ subunit (ICAP18; TGME49_268830), the putative a subunit (TGME49_310360), and the putative inhibitor (TGME49_215350). We have also identified other mitochondrial domains among the novel subunits. TGME49_258060 and TGME49_285510 bear homology to $\mathrm{CHCH}$ domain-containing proteins, which localize to the mitochondrial intermembrane space and play roles in biogenesis and bioenergetics (Modjtahedi, Tokatlidis, Dessen, \& Kroemer, 2016). ICAP15 has a low-probability Bcl-like domain, common among proteins involved in apoptosis and regulation of the electron transport chain (Gross \& Katz, 2017). Combination of conserved and phylum-specific subunits also characterize the ATP synthase complexes of other protists (Balabaskaran Nina et al., 2010; Šubrtová et al., 2015; van Lis et al., 2007). Our work demonstrates that distant homology searches can complement the molecular characterization of protein complexes in apicomplexans as in other divergent eukaryotes.

The length and extraneous domains present in ICAP2 make it a highly unusual $b$ subunit, which has motivated the characterization we present. In particular, ICAP2 is nearly twice as long as its 
mammalian counterparts and appears to have an EF-like domain in its $\mathrm{N}$ terminus. EF domains bind cations like $\mathrm{Ca}^{2+}$ or $\mathrm{Mg}^{2+}$ and can act to modulate protein-protein interactions or enzymatic activity (Kawasaki \& Kretsinger, 2017). The mitochondrial matrix is known to act as a $\mathrm{Ca}^{2+}$ store, and the presence of an EF-like domain suggests that ATP synthase activity may be somehow coordinated with mitochondrial $\mathrm{Ca}^{2+}$. Treatment of $T$. gondii with the ATP synthase inhibitor oligomycin causes an immediate increase in cytosolic Ca ${ }^{2+}$ levels (Moreno \& Zhong, 1996). Although this may simply reflect the use of ATP by the endoplasmic-reticulum $\mathrm{Ca}^{2+}$-ATPase, it also suggests a need for strong coupling between ATP production and $\mathrm{Ca}^{2+}$ sequestration. Divergent stator paralogs are expressed in the Drosophila testis and underlie tissue specific properties of the ATP synthase that impact mitochondrial morphology and activity (Sawyer et al., 2017). Having provided strong evidence for the role of ICAP2 in the ATP synthase, more work will be needed to characterize the structure of the complex, and understand how its unusual features mediate the specific adaptations of apicomplexan mitochondria.

ICAP2 is one of 11 novel subunits we believe are part of the apicomplexan ATP synthase. Orthologs of all of these subunits are found exclusively among apicomplexans, including the closest free-living ancestor Chromera velia, although they are absent from the genomes of species with reduced mitochondrial pathways. Several Cryptosporidium spp. (C. hominis, $C$. parvum, and C. ubiquitum) exhibit extreme reductions in the TCA cycle and electron transport chains, and concomitantly lack most of the known subunits of the ATP synthase along with the novel subunits we have identified (Liu et al., 2016). However, all Cryptosporidium spp. retain the $a$ and $\beta$ subunits, which could still convert $A$ TP $^{4-}$ to $A D P^{3-}$ fueling the generation of a membrane potential $(\Delta \psi)$ across the inner mitochondrial membrane through the action of the ADP/ATP carrier (AAC). A similar mechanism has been proposed for the $F_{0}$-independent generation of $\Delta \psi$ in Trypanosoma brucei strains carrying mutations in the $\gamma$ subunit (Dean, Gould, Dewar, \& Schnaufer, 2013). Even in the absence of an ATP synthase, $\Delta \psi$ is needed for the import of proteins into the matrix of mitochondria and mitosomes (Fox, 2012). Intriguingly, the genome of Gregarina niphandrodes, a divergent apicomplexan that infects beetles, lacks all of the mentioned ATP synthase subunits including $a$ and $\beta$ along with homologs of the AAC (CryptoDB), and would require an alternative mechanism to generate $\Delta \psi$ if mitosomes are present and active in this organism.

We present the first direct evidence for the important role of the ATP synthase in T. gondii metabolism. Pharmacological and metabolomics approaches had previously demonstrated the presence of ATP synthase activity in T. gondii mitochondria, and the importance of oxidative phosphorylation in supplying ATP to the parasite (MacRae et al., 2012; Vercesi et al., 1998). Consistent with these observations, depletion of ICAP2 reduces the rate of parasite replication and ultimately leads to a loss of parasite viability. This is in contrast to the mild effect of knocking out the $\beta$ subunit in blood stages of $P$. berghei, although such mutants are subsequently impaired during the sexual stages of the life cycle (Sturm et al., 2015). The variable impact of the ATP synthase on the various stages of different apicomplexans may reflect the ability of these parasites to adapt to the changing environmental conditions they encounter across their life cycles.

ICAP2 depletion appears to damage T. gondii mitochondria leading to fragmentation and loss of volume. In other eukaryotes, mitochondrial fission segregates damaged parts of the organelle (Youle \& van der Bliek, 2012). Mitochondrial fragmentation has been previously observed in T. gondii in response to exogenous stresses, including starvation (Ghosh, Walton, Roepe, \& Sinai, 2012) or monensin treatment (Charvat \& Arrizabalaga, 2016). The effect of monensin was 
attributed to its uncoupling activity and to the induction of oxidative stress. Both effects could resemble defects in the ATP synthase like those predicted to result from loss of the stator: uncoupling would dissipate the $\Delta \psi$ used by the complex, and mutations in the $F_{0}$ subunits can lead to increased reactive oxygen species (Baracca et al., 2007). Saccharomyces cerevisiae stator subunit mutants have punctate, rounded mitochondria that display an altered ultrastructure (Bornhövd et al., 2006; Weimann et al., 2008). These defects result from the involvement of the stator subunits in cristae formation (Cogliati, Enriquez, \& Scorrano, 2016; Wittig \& Schägger, 2008), and the uncoupling of ATP hydrolysis from proton translocation (Paul, Velours, Arselin de Chateaubodeau, Aigle, \& Guerin, 1989; Soubannier et al., 2002). Although ICAP2-depleted mitochondria did not show the smooth concentric membranes seen in $S$. cerevisiae stator mutants, they did have fewer cristae than wild-type mitochondria. To further understand the effects that lead to mitochondrial fragmentation, it will be interesting to compare uncoupling mutations, like ICAP2 loss, to disruption of the catalytic or proton-transporting functions of the T. gondii ATP synthase.

Without a functioning ATP synthase, the ETC is presumably inhibited by a buildup of $\Delta \psi$, which would normally be dissipated through the rotating $F_{0}$ (Mueller, 2000). We observed that the $\mathrm{mOCR}$ is reduced in ICAP2-depleted parasites, and can be restored by the addition of a proton ionophore that dissipates $\Delta \psi$. In the accompanying paper by Seidi, Muellner-Wong, and Rajendran, et al., disruption of a novel apicomplexan component of the ETC complex IV also reduced the mOCR, although this effect could not be reversed by proton ionophore treatment. This indicates a general ETC defect in the complex IV mutant distinct from that observed upon ICAP2 depletion. Notably, mutations in complex IV appear to have a milder effect on parasite viability and did not alter mitochondrial morphology. We also observed normal morphology upon ETC inhibition with atovaquone (a complex III inhibitor) and fragmented mitochondria following ATP synthase inhibition with oligomycin (data not shown). Together, these data indicate that the defects in mitochondrial morphology upon ICAP2 knockdown are not the result of general defects in the ETC, but rather a specific effect of ATP synthase impairment. In mammalian cells, inhibition of complexes I, III, or V (ATP synthase) resulted in distinct metabolic signatures (W. W. Chen, Freinkman, Wang, Birsoy, \& Sabatini, 2016). Similarly, in blood-stage malaria, it appears that the main purpose of the ETC is the regeneration of ubiquinone for pyrimidine biosynthesis (Painter, Morrisey, Mather, \& Vaidya, 2007). Therefore, inhibiting oxidative phosphorylation at different steps is expected to have different consequences, which should be investigated further as we explore these pathways as therapeutic targets in apicomplexans.

Our work identifies novel and highly divergent features of the apicomplexan ATP synthase, including two proteins that we believe comprise the complex's stator-ICAP2 and ICAP18. Previous studies had hypothesized the existence of a divergent stator in apicomplexans, and our observations confirm the presence and identity of such elements. We demonstrate the importance of these features by knocking down ICAP2, which leads to breakdown of the complex, defects in mitochondrial function and morphology, and loss of parasite viability. The conservation of these proteins in other apicomplexan species will also motivate their consideration as therapeutic targets. Future studies into the structure and function of these divergent features will help us understand their contributions to apicomplexan adaptation. Unlike most organisms, all of the ATP synthase subunits are encoded in the nuclear genome, making T. gondii a highly tractable organism to study the evolution of this important protein complex. 


\section{MATERIAL \& METHODS}

\section{Parasite culture and strains}

T. gondii tachyzoites from the strain $\mathrm{RH}$ and its derivatives were maintained at $37^{\circ} \mathrm{C}$ with $5 \%$ $\mathrm{CO}_{2}$ growing in human foreskin fibroblasts (HFFs) cultured in DMEM supplemented with $3 \%$ heat-inactivated fetal bovine serum and $10 \mu \mathrm{g} / \mathrm{ml}$ gentamicin. Parasites were transfected as described previously (Sidik, Hackett, Tran, Westwood, \& Lourido, 2014). For selection, mycophenolic acid (Sigma, cat. no. M3536) and xanthine (Sigma, cat. no. X4002) were used at $50 \mu \mathrm{g} / \mathrm{ml}$ and $25 \mu \mathrm{g} / \mathrm{ml}$, respectively.

\section{ICAP2 and ICAP18 phylogeny and topology predictions}

ICAP2 and ICAP18 homologs were readily identified by BLAST searches against all sequenced apicomplexan and chromerid genomes with the exception of $C$. hominis, $C$. parvum and the gregarine G. niphandrodes (EupathDB.org). Alignment was performed using ClustalW (Larkin et al., 2007) and the phylogenetic tree was generated by neighbor-joining excluding positions with gaps. Boot-strap values were calculated for 10,000 trials. A hidden Markov model-based search of both proteins was performed for the alignment using HHpred (Zimmermann et al., 2017).Topology predictions were performed using CCTOP (Dobson, Reményi, \& Tusnády, 2015) and mitochondrial targeting signal predictions were performed using IPSORT (Bannai, Tamada, Maruyama, Nakai, \& Miyano, 2002). Subcellular localization predictions were performed with iPSORT and TargetP 1.1 (Emanuelsson, Nielsen, Brunak, \& Heijne, 2000).

\section{Plasmid construction}

To generate the ICAP2-Ty cKD strain, a 1193 bp fragment from the 3' end of the ICAP2 coding sequence was amplified for Gibson Assembly (New England Biolabs) from genomic DNA using P9 and P10. The pG152-Lic-HA-FLAG-LoxP-3'UTRSag1-HXGPRT-LoxP-U1 (a kind gift from Markus Meissner) was linearized with Pacl and the PCR product and Pacl-linearized vector were Gibson cloned to generate the pG152-ICAP2-HA-FLAG-LoxP-3'UTRSag1-HXGPRTLoxP-U1 plasmid. For simplicity, the plasmid will be referred to as pG152-ICAP2-HA. The HA tag present on this construct was then exchanged for a Ty tag by digesting it with EcoRI and Pstl and inserting an in-frame Ty epitope (P11/P12) for Gibson Assembly. The digested vector and Ty epitope were Gibson cloned to generate the pG152-ICAP2-Ty vector. This vector was then used to generate a repair template containing the ICAP2-Ty-LoxP-3'UTRSag1-HXGPRTLoxP-U1 cassette (referred to as ICAP2-Ty CKD cassette) flanked by homology regions to the $\mathrm{C}$ terminus of the ICAP2 locus using P13 and P14.

\section{Strain generation}

The ICAP2-Ty and ICAP18-Ty strains were generated by CRISPR-mediated C-terminal Ty tagging as previously described (Sidik et al., 2016). Briefly, $30 \mu \mathrm{g}$ of a repair oligonucleotide, containing an in-frame Ty epitope flanked by homology arms targeting the $\mathrm{C}$ terminus of ICAP2 or ICAP18 (see Table S2), were co-transfected with $50 \mu \mathrm{g}$ of pU6-Universal (Addgene, cat. no. 52694) carrying the appropriate sgRNA into TATi $\triangle K U 80$ parasites (Sheiner et al., 2011). Transfected parasites were cultured until their first lysis and used to infect confluent HFF monolayers grown on coverslips. Correct expression and localization of the Ty-positive ICAPs was determined 24 hours post infection by immunofluorescence microscopy. Correct 
integration of the Ty epitope into the respective ICAP locus was confirmed by sequencing and positive parasites were isolated and subcloned by limiting dilution. To generate the ICAP2-Ty cKD strain, the ICAP2-Ty cKD cassette was transfected into the DiCre $\triangle K U 80$ strain along with the pU6-Universal plasmid carrying the same sgRNA that was used for endogenous tagging of ICAP2. Parasites were selected with xanthine and mycophenolic acid and correct expression and localization of the ICAP2-Ty positive parasites was determined 24 hours post-infection by immunofluorescence microscopy. Finally, correct integration of the ICAP2-Ty cKD cassette into the ICAP2 locus was confirmed by sequencing and parasites were isolated and subcloned by limiting dilution. For live microscopy experiments, the DiCre $\triangle K U 80$ (Andenmatten et al., 2013) and ICAP2-Ty cKD strains were transfected with pT8mycSOD2(SPTP)GFPmycHX (Pino et al., 2007) and TubIMC1TdTomato-CAT plasmids. After their first lysis, dually fluorescent cells were FACS-sorted into 96-well plates to obtain single clones.

\section{Immunofluorescence microscopy and immunoblotting}

Intracellular parasites were fixed with $4 \%$ formaldehyde at $4^{\circ} \mathrm{C}$ for $15 \mathrm{~min}$ and permeabilized with $0.25 \%$ Triton X-100 in PBS for 8 min. After blocking for 10 min with a PBS solution containing $5 \%$ normal goat serum (NGS) and 5\% heat-inactivated fetal bovine serum (IFS), staining was performed with mouse anti-Ty (clone BB2) (Bastin, Bagherzadeh, Matthews, \& Gull, 1996), rabbit anti-ALD (a gift from L. David Sibley) or rabbit anti-HSP70 ( a gift from Dominique Soldati-Favre) (Pino et al., 2007). Alexa-488-conjugated goat-anti-mouse (Invitrogen, cat. no. A11029) and Alexa-594-conjugated goat-anti-rabbit (Invitrogen, cat. no. A11037) were used as secondary antibodies. Nuclei were stained with Hoechst (Santa Cruz, (Santa Cruz, cat. no. sc-394039) and coverslips were mounted in Prolong Diamond (Thermo Fisher, cat. no. P36965). Images were acquired using an Eclipse Ti epifluorescence microscope (Nikon) using the NIS elements imaging software. FIJI was used for image analysis, and Adobe Photoshop and Illustrator CC2018 were used for image processing.

\section{Immunoblotting}

For conventional immunoblotting, parasites were resuspended in lysis buffer $(50 \mathrm{mM} \mathrm{KCl,} 20$ mM HEPES pH 7.5, 2 mM MgCl, 0.1 mM EDTA, 1\% Triton X-100, Halt protease inhibitors [Thermo Fisher, cat. no. 78440]). An equal volume of 2X Laemmli buffer (4\% SDS, 20\% glycerol, $5 \%$ 2-mercaptoethanol, 0.02\% bromophenol blue, $120 \mathrm{mM}$ Tris- $\mathrm{HCl} \mathrm{pH}$ 6.8) was added, and the samples were heated to $100^{\circ} \mathrm{C}$ for 5 min prior to resolving by SDS-PAGE. After transferring the separated proteins to nitrocellulose, membranes were blotted with mouse anti-Ty or antiTUB1 (clone 12G10, Developmental Studies Hybridoma Bank at the University of lowa); and rabbit anti-ALD1, anti-HSP70, or anti-F $\beta$ (Agrisera, cat. no. AS05085). The signal was detected using 1:10,000 dilutions of IRDye 800CW-conjugated goat anti-mouse IgG and IRDye 680CWconjugated donkey anti-rabbit IgG (LI-COR Biosciences) on an Odyssey infrared imager (LICOR Biosciences). For BN-PAGE, parasites were solubilized with 4X Native PAGE Sample Buffer (Thermo Fisher Scientific) containing 2.5\% digitonin. Separated proteins were transferred to a PVDF membrane and initially probed with mouse anti-F $\beta$ (MAb 5F4) (A. L. Chen et al., 2015) but the rabbit-anti- $F_{1} \beta$ from Agrisera was eventually used. In both cases, the membrane was incubated with a 1:10,000 dilution of horseradish peroxidase-conjugated goat-anti-rabbit antibody and were developed with the ECL system (Amersham Biosciences) according to the manufacturer's instructions. 


\section{Immunoprecipitations and silver stain}

Prior to the immunoprecipitation, $60 \mu \mathrm{g}$ of Ty antibody was cross-linked to $1 \mathrm{mg}$ of Pierce Protein G Magnetic Beads (Thermo Fisher, cat. no. 88847). Approximately $2 \times 10^{8}$ parasites were resuspended in IP lysis buffer $(150 \mathrm{mM} \mathrm{NaCl}, 20 \mathrm{mM}$ Tris pH 7.5, 0.1\%SDS, 1\% Triton $\mathrm{X}-100$, Halt protease inhibitors [Thermo Fisher]), incubated for $5 \mathrm{~min}$ at $4{ }^{\circ} \mathrm{C}$ and centrifuged at $21,000 \times g$ for $5 \mathrm{~min}$. Supernatants were incubated with the Ty-magnetic beads for $1 \mathrm{~h}$ at $4^{\circ} \mathrm{C}$ and elution was carried out by adding a solution containing $150 \mathrm{ng} / \mathrm{\mu l}$ of Ty peptide diluted in IP Iysis buffer to the beads, followed by a 30 min incubation at $4^{\circ} \mathrm{C}$. Proteins were resolved by SDS-PAGE and visualized by silver stain as described in (Shevchenko, Wilm, Vorm, \& Mann, 1996).

\section{Plaque assays}

To analyze the effect of ICAP2 on plaque formation, 500 DiCre $\triangle K U 80$ or ICAP2-Ty cKD parasites per well were added to HFF monolayers in 6-well plates and treated with vehicle or rapamycin for $2 \mathrm{~h}$. All wells were washed twice with PBS, media was added and eight days later the monolayers were rinsed with PBS, fixed in 95\% ethanol for 10 min and stained with crystal violet ( $2 \%$ crystal violet, $0.8 \%$ ammonium oxalate, $20 \%$ ethanol) for $5 \mathrm{~min}$.

\section{Live microscopy and mitochondrial volume measurements}

Parental or ICAP2-Ty cKD parasites were treated with $50 \mathrm{nM}$ rapamycin or vehicle (DMSO) upon infection of HFF monolayers in glass-bottom $35 \mathrm{~mm}$ dishes (MatTek). Rapamycin was removed after $2 \mathrm{~h}$, and parasites were allowed to grow for 24 or $48 \mathrm{~h}$. Vacuoles containing one or two parasites were imaged live using an Eclipse Ti microscope (Nikon) with an enclosure heated to $37^{\circ} \mathrm{C}$, and $5 \% \mathrm{CO}_{2}$. For each vacuole, a z-stack of approximately 25 frames at 0.2 $\mu \mathrm{m}$ spacing between each slice was taken. The bottom and top slices were set so that the mitochondria were just slightly out of focus. The mitochondrial volume was calculated using MitoGraph as described (Viana et al., 2015).

\section{Transmission electron microscopy}

For ultrastructural analyses, infected HFF cells were fixed in a freshly prepared mixture of $1 \%$ glutaraldehyde (Polysciences Inc.) and 1\% osmium tetroxide (Polysciences Inc.) in 50 mM phosphate buffer at $4^{\circ} \mathrm{C}$ for $30 \mathrm{~min}$. The low osmolarity fixative was used to dilute soluble cytosolic proteins and enhance the visualization of mitochondria. Samples were then rinsed extensively in cold $\mathrm{dH}_{2} \mathrm{O}$ prior to en bloc staining with $1 \%$ aqueous uranyl acetate (Ted Pella Inc.) at $4^{\circ} \mathrm{C}$ for $3 \mathrm{~h}$. Following several rinses in $\mathrm{dH}_{2} \mathrm{O}$, samples were dehydrated in a graded series of ethanol and embedded in Eponate 12 resin (Ted Pella Inc.). Sections of $95 \mathrm{~nm}$ were cut with a Leica Ultracut UCT ultramicrotome (Leica Microsystems Inc.), stained with uranyl acetate and lead citrate, and viewed on a JEOL 1200 EX transmission electron microscope (JEOL USA Inc.) equipped with an AMT 8-megapixel digital camera and AMT Image Capture Engine V602 software (Advanced Microscopy Techniques). For cristae enumeration, images were blinded and the mitochondrial area along with the corresponding number of cristae in each area were counted using FIJI. The images were subsequently un-blinded and the values for rapamycin-treated and vehicle were compared using a Mann-Whitney test. 


\section{Mass Spectrometry}

After each immunoprecipitation experiment, bands from the SDS-PAGE gels were excised, divided into $\sim 2 \mathrm{~mm}$ squares and washed overnight in 50\% methanol in water. These were washed once more with 47.5\% methanol $5 \%$ acetic acid in water for $2 \mathrm{~h}$, dehydrated with acetonitrile and dried in a speed-vac. Reduction and alkylation of disulfide bonds was carried out by the addition of $30 \mu \mathrm{l} 10 \mathrm{mM}$ dithiothreitol (DTT) in $100 \mathrm{mM}$ ammonium bicarbonate $\left(\mathrm{NH}_{4} \mathrm{HCO}_{3}\right)$ for $30 \mathrm{~min}$. Samples were alkylated by removal of the DTT solution and addition of $100 \mathrm{mM}$ iodoacetamide in $100 \mathrm{mM} \mathrm{NH}_{4} \mathrm{HCO}_{3}$ for $30 \mathrm{~min}$. Samples were sequentially washed with aliquots of acetonitrile, $100 \mathrm{mM} \mathrm{NH}_{4} \mathrm{HCO}_{3}$ and acetonitrile and dried in a speed-vac. The bands were enzymatically digested with $300 \mathrm{ng}$ of sequencing-grade Trypsin (Promega, cat. no. V5111) in $50 \mathrm{mM} \mathrm{NH}_{4} \mathrm{HCO}_{3}$ for 10 mins, on ice. Sufficient $\mathrm{NH}_{4} \mathrm{HCO}_{3}$ was added to rehydrate the gel pieces. These were allowed to digest overnight at $37^{\circ} \mathrm{C}$ with gentle agitation. Peptides were extracted by gently agitating the gel bands 10 min with sequential $50 \mu$ l washes with 50 $\mathrm{mM} \mathrm{NH}_{4} \mathrm{HCO}_{3}$, and twice with $47.5 \%$ acetonitrile $5 \%$ formic acid in water. All extractions were pooled in a $0.5 \mathrm{ml}$ conical autosampler vial. Organic solvent was removed and the volumes were reduced to $15 \mu \mathrm{l}$ using a speed-vac. Samples were analyzed by reversed-phase highperformance liquid chromatography using a Waters NanoAcquity in line with a ThermoFisher Orbitrap Elite mass spectrometer, in a nano-flow configuration. The mass spectrometer was operated in a top 10 data-dependent acquisition mode using a 240,000 FWHM m/z resolution in the Orbitrap and fragmentation in the linear ion trap.

\section{Mass spectrometry data analysis}

Peptide searches were performed with Mascot (Matrix Science) algorithms against the T. gondii protein database (version 11, ToxoDB.org) concatenated to a database of common contaminants (keratin, trypsin, etc). The resulting Mascot search results were then loaded into Scaffold (Proteome Software) and a minimum peptide threshold of 95\% was used for identification of peptides prior to the analysis described in the results.

\section{ECAR and mOCR measurements}

Parental and ICAP2-Ty cKD parasites were grown in the absence of rapamycin, or incubated in $50 \mathrm{nM}$ rapamycin for $2 \mathrm{~h}$, and cultured for a further 1-3 days before analysis. Parasites filtered through $3 \mu \mathrm{m}$ were washed twice in Seahorse XF base medium (Agilent Technologies), supplemented with $1 \mathrm{mM}$ L-glutamine and $5 \mathrm{mM}$ D-glucose, before re-suspension to $1.5 \times 10^{7}$ cells $/ \mathrm{ml}$. $100 \mu \mathrm{l}$ of the parasite suspensions were seeded into wells of a 96-well culture plate coated with $3.5 \mu \mathrm{g} / \mathrm{cm}^{2}$ of CellTak cell adhesive (In Vitro Technologies, cat. no. FAL354240). The plate was centrifuged at $50 \times g$ for $3 \mathrm{~min}$ and an additional $75 \mu \mathrm{l}$ of base media (Agilent Technologies) was added to each well. Parasites were kept at $37^{\circ} \mathrm{C}$ in ambient air until the start of experiment. Parasite oxygen consumption rates (OCR) and extracellular acidification rate (ECAR) were measured using an Agilent Seahorse XFe96 Analyzer. To determine the maximal OCR, parasites were treated with FCCP to a final concentration of $1 \mu \mathrm{M}$. To determine the nonmitochondrial OCR, parasites were treated with $10 \mu \mathrm{M}$ Antimycin $\mathrm{A}$ and $1 \mu \mathrm{M}$ Atovaquone. OCR measurements were acquired for 3 min each, at 6 min intervals across the course of the experiment. Results were compiled using the Wave Desktop program and analysis of parasite OCR was performed using the R software environment. Linear mixed-effects models were fitted to the data, with error between plates and wells (i.e., between and within experiments) defined 
as the random effect, and the OCR measurements in the different parasite strains and the time after rapamycin treatment defined as the fixed effect. A minimum of 4 wells was used for background correction in each assay plate. Parasite mOCR was computed by subtracting the non-mitochondrial OCR measured following addition of Antimycin A and Atovaquone from the basal OCR. The spare capacity of the parasite ETC was computed by measuring the difference between basal mOCR and maximal mOCR (following the addition of FCCP).

\section{ACKNOWLEDGEMENTS}

We thank Eric Spooner at the Whitehead Proteomics Core and Wandy Beatty at the Washington University Molecular Microbiology Imaging Facility for scientific services, L. David Sibley for the ALD antibody, Peter Bradley for the $F_{1} \beta$ antibody, Dominique Soldati-Favre for the HSP70 antibody and the SOD2-GFP vector, and Markus Meissner for the DiCre $\triangle K U 80$ strain and the U1-tagging plasmid. We would also like to thank Benedikt Markus and Emily Shortt for technical support and useful discussions. This work was supported by the NIH Director's Early Independence Award (1DP5OD017892) and an NIH Exploratory R21 grant (1R21AI123746) to S.L., and an NIH Pathway to Independence Award (K99AI137218) to D.H.

\section{SUPPLEMENTARY FIGURE LEGENDS}

Figure S1. Alignment of the putative $\mathrm{Ca}^{2+-b i n d i n g ~ d o m a i n ~(g r e e n) ~ a n d ~ t h e ~ r e g i o n ~ s t r u c t u r a l l y ~}$ similar to BtATPb (blue) found in ICAP2 and its homologs in 11 different apicomplexan species. Residues that are identical to or chemically similar to the consensus amino acid are shown in a colored background.

Figure S2. Alignment of the region sharing structural similarity between BtATPd, ICAP18 and its homologs found in 11 different apicomplexans. Residues that are identical to or chemically similar to the consensus amino acid are shown with a colored background.

Figure S3. Eluted fractions from Ty immunoprecipitation experiments using the ICAP2Ty and ICAP18Ty strains separated by SDS-PAGE and visualized by silver stain. The four experiments (three with ICAP2Ty, one with ICAP18Ty) along the excised bands used for MS analysis found in Table S1 are indicated.

Figure S4. (A) Comparison of $\mathrm{mOCR}$ (pmol/min/1.5 x $10^{6}$ parasites) and ECAR $(\mathrm{mpH} / \mathrm{min} / 1.5$ $\times 10^{6}$ parasites) for parental and CKD strains following the indicated times after a $2 \mathrm{~h}$ rapa pulse.(B) Maximal mOCR obtained after treated each strain with the uncoupling agent FCCP. Data represent mean \pm SEM for $n=4$ independent experiments: n.s. not significant; one-way ANOVA followed by Tukey's test.

Table S1. Summary of mass spectrometry data showing proteins identified in ICAP2-Ty and ICAP18-Ty immunoprecipitations.

Table S2. Primers and oligonucleotides used in this study. 


\section{BIBLIOGRAPHY}

Andenmatten, N., Egarter, S., Jackson, A. J., Jullien, N., Herman, J.-P., \& Meissner, M. (2013). Conditional genome engineering in Toxoplasma gondii uncovers alternative invasion mechanisms. Nature Methods, 10(2), 125-127. http://doi.org/10.1038/nmeth.2301

Balabaskaran Nina, P., Dudkina, N. V., Kane, L. A., van Eyk, J. E., Boekema, E. J., Mather, M. W., \& Vaidya, A. B. (2010). Highly divergent mitochondrial ATP synthase complexes in Tetrahymena thermophila. PLoS Biology, 8(7), e1000418. http://doi.org/10.1371/journal. pbio. 1000418

Balabaskaran Nina, P., Morrisey, J. M., Ganesan, S. M., Ke, H., Pershing, A. M., Mather, M. W., \& Vaidya, A. B. (2011). ATP synthase complex of Plasmodium falciparum: dimeric assembly in mitochondrial membranes and resistance to genetic disruption. The Journal of Biological Chemistry, 286(48), 41312-41322. http://doi.org/10.1074/jbc.M111.290973

Bannai, H., Tamada, Y., Maruyama, O., Nakai, K., \& Miyano, S. (2002). Extensive feature detection of N-terminal protein sorting signals. Bioinformatics, 18(2), 298-305. http://doi. org/10.1093/bioinformatics/18.2.298

Baracca, A., Sgarbi, G., Mattiazzi, M., Casalena, G., Pagnotta, E., Valentino, M. L., et al. (2007). Biochemical phenotypes associated with the mitochondrial ATP6 gene mutations at nt8993. Biochimica Et Biophysica Acta (BBA) - Bioenergetics, 1767(7), 913-919. http://doi. org/10.1016/j.bbabio.2007.05.005

Bastin, P., Bagherzadeh, Z., Matthews, K. R., \& Gull, K. (1996). A novel epitope tag system to study protein targeting and organelle biogenesis in Trypanosoma brucei. Molecular and Biochemical Parasitology, 77(2), 235-239.

Behnke, M. S., Wootton, J. C., Lehmann, M. M., Radke, J. B., Lucas, O., Nawas, J., et al. (2010). Coordinated progression through two subtranscriptomes underlies the tachyzoite cycle of Toxoplasma gondii. PLOS ONE, 5(8), e12354. http://doi.org/10.1371/journal. pone.0012354

Boratyn, G. M., Camacho, C., Cooper, P. S., Coulouris, G., Fong, A., Ma, N., et al. (2013). BLAST: a more efficient report with usability improvements. Nucleic Acids Research, 41(W1), W29-W33. http://doi.org/10.1093/nar/gkt282

Bornhövd, C., Vogel, F., Neupert, W., \& Reichert, A. S. (2006). Mitochondrial membrane potential is dependent on the oligomeric state of $F_{1} F_{0}$-ATP synthase supracomplexes. Journal of Biological Chemistry, 281(20), 13990-13998. http://doi.org/10.1074/jbc. M512334200

Charvat, R. A., \& Arrizabalaga, G. (2016). Oxidative stress generated during monensin treatment contributes to altered Toxoplasma gondii mitochondrial function. Scientific Reports, 6(1), 22997. http://doi.org/10.1038/srep22997

Chen, A. L., Kim, E. W., Toh, J. Y., Vashisht, A. A., Rashoff, A. Q., Van, C., et al. (2015). Novel components of the Toxoplasma inner membrane complex revealed by BiolD. mBio, 6(1), e02357-14. http://doi.org/10.1128/mBio.02357-14

Chen, W. W., Freinkman, E., Wang, T., Birsoy, K., \& Sabatini, D. M. (2016). Absolute Quantification of Matrix Metabolites Reveals the Dynamics of Mitochondrial Metabolism. Cell, 166(5), 1324-1337.e11. http://doi.org/10.1016/j.cell.2016.07.040 
Cogliati, S., Enriquez, J. A., \& Scorrano, L. (2016). Mitochondrial Cristae: Where Beauty Meets Functionality. Trends in Biochemical Sciences, 41(3), 261-273. http://doi.org/10.1016/j. tibs.2016.01.001

Dean, S., Gould, M. K., Dewar, C. E., \& Schnaufer, A. C. (2013). Single point mutations in ATP synthase compensate for mitochondrial genome loss in trypanosomes. Proceedings of the National Academy of Sciences of the United States of America, 110(36), 14741-14746. http://doi.org/10.1073/pnas. 1305404110

Denton, H., Roberts, C. W., Alexander, J., Thong, K. W., \& Coombs, G. H. (1996). Enzymes of energy metabolism in the bradyzoites and tachyzoites of Toxoplasma gondii. FEMS Microbiology Letters, 137(1), 103-108.

Dickson, V. K., Silvester, J. A., Fearnley, I. M., Leslie, A. G. W., \& Walker, J. E. (2006). On the structure of the stator of the mitochondrial ATP synthase. The EMBO Journal, 25(12), 29112918. http://doi.org/10.1038/sj.emboj.7601177

Dobson, L., Reményi, I., \& Tusnády, G. E. (2015). CCTOP: a Consensus Constrained TOPology prediction web server. Nucleic Acids Research, 43(W1), W408-W412. http://doi. org/10.1093/nar/gkv451

Emanuelsson, O., Nielsen, H., Brunak, S., \& Heijne, von, G. (2000). Predicting Subcellular Localization of Proteins Based on their N-terminal Amino Acid Sequence. Journal of Molecular Biology, 300(4), 1005-1016. http://doi.org/10.1006/jmbi.2000.3903

Fox, T. D. (2012). Mitochondrial protein synthesis, import, and assembly. Genetics, 192(4), 1203-1234. http://doi.org/10.1534/genetics.112.141267

Ghosh, D., Walton, J. L., Roepe, P. D., \& Sinai, A. P. (2012). Autophagy is a cell death mechanism in Toxoplasma gondii. Cellular Microbiology, 14(4), 589-607. http://doi. org/10.1111/j.1462-5822.2011.01745.x

Gross, A., \& Katz, S. G. (2017). Non-apoptotic functions of BCL-2 family proteins. Cell Death and Differentiation, 24(8), 1348-1358. http://doi.org/10.1038/cdd.2017.22

He, J., Carroll, J., Ding, S., Fearnley, I. M., \& Walker, J. E. (2017). Permeability transition in human mitochondria persists in the absence of peripheral stalk subunits of ATP synthase. Proceedings of the National Academy of Sciences, 114(34), 9086-9091. http://doi. org/10.1073/pnas. 1711201114

Jonckheere, A. I., Smeitink, J. A. M., \& Rodenburg, R. J. T. (2012). Mitochondrial ATP synthase: architecture, function and pathology. Journal of Inherited Metabolic Disease, 35(2), 211-225. http://doi.org/10.1007/s10545-011-9382-9

Kane, L. A., Youngman, M. J., Jensen, R. E., \& van Eyk, J. E. (2010). Phosphorylation of the $F(1) F(0)$ ATP synthase beta subunit: functional and structural consequences assessed in a model system. Circulation Research, 106(3), 504-513. http://doi.org/10.1161/ CIRCRESAHA.109.214155

Kawasaki, H., \& Kretsinger, R. H. (2017). Structural and functional diversity of EF-hand proteins: Evolutionary perspectives. Protein Science, 26(10), 1898-1920. http://doi. org/10.1002/pro.3233

Lapaille, M., Escobar-Ramírez, A., Degand, H., Baurain, D., Rodríguez-Salinas, E., Coosemans, N., et al. (2010). Atypical subunit composition of the chlorophycean 
mitochondrial $F_{1} F_{0}$-ATP synthase and role of Asa7 protein in stability and oligomycin resistance of the enzyme. Molecular Biology and Evolution, 27(7), 1630-1644. http://doi. org/10.1093/molbev/msq049

Larkin, M. A., Blackshields, G., Brown, N. P., Chenna, R., McGettigan, P. A., McWilliam, H., et al. (2007). Clustal W and Clustal X version 2.0. Bioinformatics, 23(21), 2947-2948. http://doi. org/10.1093/bioinformatics/btm404

Liu, S., Roellig, D. M., Guo, Y., Li, N., Frace, M. A., Tang, K., et al. (2016). Evolution of mitosome metabolism and invasion-related proteins in Cryptosporidium. BMC Genomics, 17(1), 1-16. http://doi.org/10.1186/s12864-016-3343-5

MacRae, J. I., Dixon, M. W., Dearnley, M. K., Chua, H. H., Chambers, J. M., Kenny, S., et al. (2013). Mitochondrial metabolism of sexual and asexual blood stages of the malaria parasite Plasmodium falciparum. BMC Biology, 11(1), 67. http://doi.org/10.1186/1741-7007-11-67

MacRae, J. I., Sheiner, L., Nahid, A., Tonkin, C., Striepen, B., \& McConville, M. J. (2012). Mitochondrial metabolism of glucose and glutamine is required for intracellular growth of Toxoplasma gondii. Cell Host and Microbe, 12(5), 682-692. http://doi.org/10.1016/j. chom.2012.09.013

Makiuchi, T., \& Nozaki, T. (2014). Highly divergent mitochondrion-related organelles in anaerobic parasitic protozoa. Biochimie, 100(C), 3-17. http://doi.org/10.1016/j. biochi.2013.11.018

Mezulis, S., Yates, C. M., Wass, M. N., Sternberg, M. J. E., \& Kelley, L. A. (2015). The Phyre2 web portal for protein modeling, prediction and analysis. Nature Protocols, 10(6), 845-858. http://doi.org/10.1038/nprot.2015-053

Modjtahedi, N., Tokatlidis, K., Dessen, P., \& Kroemer, G. (2016). Mitochondrial Proteins Containing Coiled-Coil-Helix-Coiled-Coil-Helix $(\mathrm{CHCH})$ Domains in Health and Disease. Trends in Biochemical Sciences, 41(3), 245-260. http://doi.org/10.1016/j.tibs.2015.12.004

Moreno, S. N., \& Zhong, L. (1996). Acidocalcisomes in Toxoplasma gondii tachyzoites. The Biochemical Journal, 313 ( Pt 2)(Pt 2), 655-659.

Mueller, D. M. (2000). Partial assembly of the yeast mitochondrial ATP synthase. Journal of Bioenergetics and Biomembranes, 32(4), 391-400.

Nishi, M., Hu, K., Murray, J. M., \& Roos, D. S. (2008). Organellar dynamics during the cell cycle of Toxoplasma gondii. Journal of Cell Science, 121(Pt 9), 1559-1568. http://doi. org/10.1242/jcs.021089

Ovciarikova, J., Lemgruber, L., Stilger, K. L., Sullivan, W. J., \& Sheiner, L. (2017). Mitochondrial behaviour throughout the lytic cycle of Toxoplasma gondii. Scientific Reports, 7, 1-13. http://doi.org/10.1038/srep42746

Painter, H. J., Morrisey, J. M., Mather, M. W., \& Vaidya, A. B. (2007). Specific role of mitochondrial electron transport in blood-stage Plasmodium falciparum. Nature, 446(7131), 88-91. http://doi.org/10.1038/nature05572

Paul, M. F., Velours, J., Arselin de Chateaubodeau, G., Aigle, M., \& Guerin, B. (1989). The role of subunit 4, a nuclear-encoded protein of the F0 sector of yeast mitochondrial ATP synthase, in the assembly of the whole complex. European Journal of Biochemistry, 185(1), 163-171. 
Perez, E., Lapaille, M., Degand, H., Cilibrasi, L., Villavicencio-Queijeiro, A., Morsomme, P., et al. (2014). The mitochondrial respiratory chain of the secondary green alga Euglena gracilis shares many additional subunits with parasitic Trypanosomatidae. Mitochondrion, 19 Pt B, 338-349. http://doi.org/10.1016/j.mito.2014.02.001

Pieperhoff, M. S., Pall, G. S., Jiménez-Ruiz, E., Das, S., Melatti, C., Gow, M., et al. (2015). Conditional U1 Gene Silencing in Toxoplasma gondii. PLOS ONE, 10(6), e0130356. http:// doi.org/10.1371/journal.pone.0130356

Pino, P., Aeby, E., Foth, B. J., Sheiner, L., Soldati, T., Schneider, A., \& Soldati-Favre, D. (2010). Mitochondrial translation in absence of local tRNA aminoacylation and methionyl tRNA Met formylation in Apicomplexa. Molecular Microbiology, 76(3), 706-718. http://doi. org/10.1111/j.1365-2958.2010.07128.x

Pino, P., Foth, B. J., Kwok, L.-Y., Sheiner, L., Schepers, R., Soldati, T., \& Soldati-Favre, D. (2007). Dual targeting of antioxidant and metabolic enzymes to the mitochondrion and the apicoplast of Toxoplasma gondii. PLoS Pathogens, 3(8), e115. http://doi.org/10.1371/ journal.ppat.0030115

Sawyer, E. M., Brunner, E. C., Hwang, Y., Ivey, L. E., Brown, O., Bannon, M., et al. (2017). Testis-specific ATP synthase peripheral stalk subunits required for tissue-specific mitochondrial morphogenesis in Drosophila, 1-15. http://doi.org/10.1186/s12860-017-0132-1

Sheiner, L., Demerly, J. L., Poulsen, N., Beatty, W. L., Lucas, O., Behnke, M. S., et al. (2011). A systematic screen to discover and analyze apicoplast proteins identifies a conserved and essential protein import factor. PLoS Pathogens, 7(12), e1002392. http://doi.org/10.1371/ journal.ppat. 1002392

Shevchenko, A., Wilm, M., Vorm, O., \& Mann, M. (1996). Mass Spectrometric Sequencing of Proteins from Silver-Stained Polyacrylamide Gels. Analytical Chemistry, 68(5), 850-858. http://doi.org/10.1021/ac950914h

Sidik, S. M., Hackett, C. G., Tran, F., Westwood, N. J., \& Lourido, S. (2014). Efficient Genome Engineering of Toxoplasma gondii Using CRISPR/Cas9. PLOS ONE, 9(6), e100450. http:// doi.org/10.1371/journal.pone.0100450.s003

Sidik, S. M., Huet, D., Ganesan, S. M., Huynh, M.-H., Wang, T., Nasamu, A. S., et al. (2016). A Genome-wide CRISPR Screen in Toxoplasma Identifies Essential Apicomplexan Genes. Cell, 166(6), 1-26. http://doi.org/10.1016/j.cell.2016.08.019

Soubannier, V., Vaillier, J., Paumard, P., Coulary, B., Schaeffer, J., \& Velours, J. (2002). In the absence of the first membrane-spanning segment of subunit 4(b), the yeast ATP synthase is functional but does not dimerize or oligomerize. Journal of Biological Chemistry, 277(12), 10739-10745. http://doi.org/10.1074/jbc.M111882200

Sturm, A., Mollard, V., Cozijnsen, A., Goodman, C. D., \& McFadden, G. I. (2015). Mitochondrial ATP synthase is dispensable in blood-stage Plasmodium berghei rodent malaria but essential in the mosquito phase. Proceedings of the National Academy of Sciences of the United States of America, 112(33), 10216-10223. http://doi.org/10.1073/ pnas. 1423959112 
Sun, M., Li, W., Blomqvist, K., Das, S., Hashem, Y., Dvorin, J. D., \& Frank, J. (2015).

Dynamical features of the Plasmodium falciparum ribosome during translation. Nucleic Acids Research, 43(21), 10515-10524. http://doi.org/10.1093/nar/gkv991

Šubrtová, K., Panicucci, B., \& Zíková, A. (2015). ATPaseTb2, a Unique Membrane-bound $\mathrm{F}_{0} \mathrm{~F}_{1}$-ATPase Component, Is Essential in Bloodstream and Dyskinetoplastic Trypanosomes. PLoS Pathogens, 11(2), e1004660-27. http://doi.org/10.1371/journal.ppat.1004660

Uboldi, A. D., McCoy, J. M., Blume, M., Gerlic, M., Ferguson, D. J. P., Dagley, L. F., et al. (2015). Regulation of Starch Stores by a Ca(2+)-Dependent Protein Kinase Is Essential for Viable Cyst Development in Toxoplasma gondii. Cell Host and Microbe, 18(6), 670-681. http://doi.org/10.1016/j.chom.2015.11.004

Vaidya, A. B., \& Mather, M. W. (2009). Mitochondrial Evolution and Functions in Malaria Parasites. Annual Review of Microbiology, 63(1), 249-267. http://doi.org/10.1146/annurev. micro.091208.073424

van Lis, R., Mendoza-Hernández, G., Groth, G., \& Atteia, A. (2007). New insights into the unique structure of the $F_{0} F_{1}$-ATP synthase from the chlamydomonad algae Polytomella sp. and Chlamydomonas reinhardtii. Plant Physiology, 144(2), 1190-1199. http://doi. org/10.1104/pp. 106.094060

Vercesi, A. E., Rodrigues, C. O., Uyemura, S. A., Zhong, L., \& Moreno, S. N. (1998).

Respiration and oxidative phosphorylation in the apicomplexan parasite Toxoplasma gondii. Journal of Biological Chemistry, 273(47), 31040-31047.

Viana, M. P., Lim, S., \& Rafelski, S. M. (2015). Quantifying mitochondrial content in living cells. Biophysical Methods in Cell Biology (Vol. 125, pp. 77-93). Elsevier Ltd. http://doi. org/10.1016/bs.mcb.2014.10.003

Walker, J. E. (2013). The ATP synthase: the understood, the uncertain and the unknown. Biochemical Society Transactions, 41(1), 1-16. http://doi.org/10.1042/BST20110773

Weimann, T., Vaillier, J., Salin, B., \& Velours, J. (2008). The Intermembrane Space Loop of Subunit b( 4) Is a Major Determinant of the Stability of Yeast Oligomeric ATP Synthases $\dagger$. Biochemistry, 47(11), 3556-3563. http://doi.org/10.1021/bi702000g

Wittig, I., \& Schägger, H. (2008). Structural organization of mitochondrial ATP synthase. Biochimica Et Biophysica Acta, 1777(7-8), 592-598. http://doi.org/10.1016/j. bbabio.2008.04.027

Youle, R. J., \& van der Bliek, A. M. (2012). Mitochondrial fission, fusion, and stress. Science, 337(6098), 1062-1065. http://doi.org/10.1126/science.1219855

Zimmermann, L., Stephens, A., Nam, S.-Z., Rau, D., Kübler, J., Lozajic, M., et al. (2017). A Completely Reimplemented MPI Bioinformatics Toolkit with a New HHpred Server at its Core. Journal of Molecular Biology, 1-7. http://doi.org/10.1016/j.jmb.2017.12.007 